\title{
Adaptive Sliding Mode Guidance Law with Terminal Angle Constraint
}

\author{
Zhang Kuanqiao* \\ Missile Engineering Department \\ Shijiazhuang Mechanical Engineering College \\ Shijiazhuang, China \\ zkuanqiao@163.com \\ * Corresponding Author
}

\author{
Yang Suochang \\ Missile Engineering Department \\ Shijiazhuang Mechanical Engineering College \\ Shijiazhuang, Ch ina \\ Yangsuochang_jx@sina.com
}

\begin{abstract}
As it is important to attack the targets with terminal angle constraint for a lot of guided weapons, an adaptive sliding mode guidance law is proposed based on the sliding mode variable structure control theory. Considering the problems that the terminal line of sight angle speed changes rapidly and terminal angle constraint, the guidance law chooses relative velocity deflection angle as the sliding mode surface, introduces terminal angle constraint. regards the target maneuvering as limited disturbance, and uses Lya punov stability theory to analyze the stability of the guidance law. In order to weaken the chattering phenomenon of variable structure control, the guidance law proposes a new method that combining the variable switch term with saturated function. Finallv. the designed guidance law was applied to the missile actual model to make a contrastive simulation, the results show that compared with the traditional method, the proposed guidance law can attack the target with less miss distance and terminal angle error, and has strong robustness.
\end{abstract}

Keywords-guidance law; terminal angle constraint; sliding mode variable structure; relative velocity deflection angle; adaptive reaching law

\section{INT RODUCTION}

In the modern wars, how to improve the accuracy and damage effectiveness of guided weapons is always a hot issue for many domestic and overseas scholars. For a lot of guided weapons, such as anti-tank missiles and anti-ship missiles, not only want to attack the targets with high accuracy, but also hope to hit the target with definite terminal impact angle, so the design of the guidance law needs to consider the problem of impact angle constraint [1].

Since Kim and Grider introduced the terminal impact angle constraint into the guidance system of the ballistic missile reentry vehicles for the first time, it has already gone through 40 years for the research of guidance law with impact angle constraint, and emerged many results, such as the optimal guidance law [2-4], the improved proportional guidance law [5-7], the geo metry curve guidance law [8-9], etc. The optimal guidance law is not restricted by performance indicators and terminal constraints, and has the best guidance performance in the ideal situation, but it relies on various assumptions and simplifications, and has poor robustness; The improve proportional guidance law has simple structure, does not depend on the information of the range and velocity, and it is easy to implement, but it relies on highly accurate guidance information, so its anti-interference ability is poor; The geometry curve guidance law does not need the distance information, but relies on various assumptions and simplifications, its guidance precision is not high.

Due to the sliding mode variable structure control has strong adaptability and robustness with the parameter perturbation and external disturbance, and its algorithm is relatively simple, variab le structure control has been gradually applied to the design of guidance law. By bringing terminal angle constraint into the sliding mode, many literatures have deduced the variable structure guidance laws with terminal angle constraints [10-12]. Most of the literatures selected the line of sight angular rate or the product of the line of sight angle rate and the missile-target relative distance as the sliding mode surface, at the same time introduce the terminal angular constraint, but with the decrease of the relative distance, the line of sight angular rate will change rapidly, that will cause the missile attitude changes rapidly in the end of the terminal, and the stability is poor. Considering this problem, a variable structure guidance law was proposed based on the relative velocity deflection angle [13-14]. It chose the relative velocity deflection angle as sliding mode surface, but did not consider the terminal angle constraint. In this paper, aiming at the terminal angle constraint problem, we select the relative velocity angle and terminal angle constraint term as the sliding mode surface, establish the missile-target relative motion model, regard the target maneuvering as a bounded disturbance, and propose an adaptive sliding mode guidance law with terminal angle constraint. Finally, the guidance performance is tested by the contrastive simulations.

\section{MISSILE-TO-TARGET MOTION MODEL}

In front of designing the guidance law, the missile-target relative motion model is established first, as shown in Figure 1.

In the Fig .1, $M$ is the missile, $T$ represents the target. Where $v_{m}$ is the missile's speed, $\theta_{m}$ is the missile's elevation angle, $a_{m}$ is the missile's acceleration; $v_{t}$ is target's speed, $\theta_{t}$ is the tracking angle, $a_{t}$ is the target's acceleration; $r$ is the distance between the missile and the target, $q$ is the line 
of sight angle, $v_{r}$ is the missile-target relative velocity which means $\vec{v}_{r}=\vec{v}_{m}-\vec{v}_{t}, a_{r}$ is the relative acceleration, $\eta_{r}$ is the relative velocity deflection angle which means the angle between the relative velocity and the line of sight.

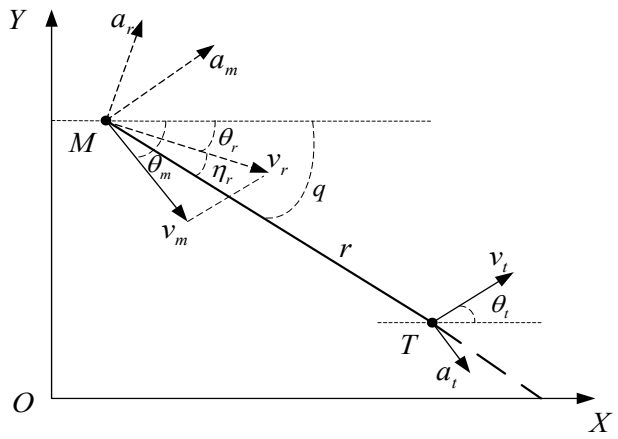

Figure 1. Missile-to-target motion model

Based on the missile-to-target relative motion relation, the relative motion equations are as follows:

$$
\left\{\begin{array}{l}
\dot{r}=-v_{m} \cos \left(q-\theta_{m}\right)+v_{t} \cos \left(q-\theta_{t}\right) \\
r \dot{q}=v_{m} \sin \left(q-\theta_{m}\right)-v_{t} \sin \left(q-\theta_{t}\right) \\
a_{m}=v_{m} \dot{\theta}_{m} \\
a_{t}=v_{t} \dot{\theta}_{t} \\
a_{r}=v_{r} \dot{\theta}_{r}
\end{array}\right.
$$

\section{DESIGN AND ANALYSIS OF THE GUIDANCE LAW}

\section{A. Derivation of The Guidance Law}

From the Fig. 1, $\eta_{r}$ can be described as

$$
\eta_{r}=q-\theta_{r}
$$

While $q$ is equal to $\theta_{r}$ which means $\eta_{r}$ is zero, it can be ensured that the missile hits the target precisely, and $\eta_{r}$ will not like $q$ that changes rapidly with the decrease of the missile-target relative distance, so selecting $\eta_{r}$ as the sliding mode surface, not only can solve the problem that $q$ changes sharply at the end of guidance, but also can guide the missile attacking the target with quasi-parallel approaching method. Using this method, the ballistic curve will be smoother, and the requirement of the control system is relatively low. Considering the terminal angle constraint, the switching function of the sliding mode is selected as follows:

$$
s=\eta_{r}+k_{1}\left(q-q_{d}\right)
$$

Where $k_{1}>0, q_{d}$ is the expected terminal angle. The first term of the switching function makes the relative velocity deflection angle tend to zero to make sure that the missile can hit the target; the second term is used to satisfy the terminal angle constraint.

After selecting the sliding mode surface, we should choose the reaching law. The commonly used reaching laws mainly include constant reaching law, exponential reaching law, and power reaching law. By taking account of time variation of the control system, and ensuring the reaching condition and good dynamic performance, an adaptive exponential reaching law [15] is chosen. Its expression is as follows:

$$
\dot{s}=\frac{k_{2} \dot{r}}{r} s-\frac{\varepsilon}{r} \operatorname{sgn}(s)
$$

Where $k_{2}>0, \varepsilon>0$. At the beginning of the terminal guidance phase, $r$ is bigger, that makes the approach speed slower, so the guidance instruction is relatively suitable, that can effectively avoid the guidance commands saturated, and reduce the missile's required overload. With $r$ approaching to zero, the approach speed increased rapidly, that urges $s$ to converge to zero to avoid divergence. When $s$ tend to be zero, the reaching rate is about $\varepsilon / r$, that can guarantee the guidance law to reach the sliding mode surface with limited time.

From the Fig. 1, we can gain the equations as follows:

$$
\begin{gathered}
a_{r}=a_{m} \cos \left(q-\eta_{r}-\theta_{m}\right)-a_{t} \cos \left(q-\eta_{r}-\theta_{t}\right) \\
a_{r}=v_{r} \dot{\theta}_{r}=v_{r}\left(\dot{q}-\dot{\eta}_{r}\right)
\end{gathered}
$$

With equation (6) and (7), we can gain

$$
\dot{\eta}_{r}=\dot{q}-\frac{a_{m} \cos \left(q-\eta_{r}-\theta_{m}\right)-a_{t} \cos \left(q-\eta_{r}-\theta_{t}\right)}{v_{r}}
$$

Differentiating Eq. (3), we can obtain the following equation:

$$
\dot{s}=\dot{\eta}_{r}+k_{1} \dot{q}
$$

Substituting Eq. (7) into Eq. (8) and combining with Eq. (4) gives

$$
\begin{aligned}
& \frac{k_{2} \dot{r}}{r} s-\frac{\varepsilon}{r} \operatorname{sgn}(s)=\left(k_{1}+1\right) \dot{q}- \\
& \frac{a_{m} \cos \left(q-\eta_{r}-\theta_{m}\right)-a_{t} \cos \left(q-\eta_{r}-\theta_{t}\right)}{v_{r}}
\end{aligned}
$$

In the process of the missile attacking the target, $\dot{r}$ changes inconspicuously, so we can assume that $\ddot{r}$ is zero. With the above equation the missile's acceleration can be described as follows:

$$
\begin{aligned}
a_{c}= & a_{m}=\frac{1}{\cos \left(q-\eta_{r}-\theta_{m}\right)}\left[\left(k_{1}+1\right) v_{r} \dot{q}+\right. \\
& \left.a_{t} \cos \left(q-\eta_{r}-\theta_{t}\right)-v_{r} \frac{k_{2} \dot{r} s-\varepsilon \operatorname{sgn}(s)}{r}\right]
\end{aligned}
$$

It is difficult to obtain the information of the target's maneuvering and position, while the missile attacks the target. Kalman Filter is commonly used to solve the problem, but it will cause larger error. So Eq. (10) is hard to be calculated. As a matter of fact, the target's velocity and maneuvering are much less than the missile, and the target's maneuvering is limited, so we can assume that $\left|a_{\mathrm{t}}\right| \leq M$, where $M$ is the 
maximum value of the target's acceleration, then $a_{t} \cos \left(q-\eta_{r}-\theta_{t}\right) \leq M$. Based on the robustness of variable structure control against the external disturbance, simplify ing Eq. (10) can obtain the adaptive sliding mode variable structure guidance law with terminal angle constraint as follows:

$$
a_{c}=\frac{1}{\cos \left(q-\eta_{r}-\theta_{m}\right)}\left[\left(k_{1}+1\right) v_{r} \dot{q}-v_{r} \frac{k_{2} \dot{r} s-\varepsilon \operatorname{sgn}(s)}{r}\right]
$$

Where $\dot{q}, r$ and $\dot{r}$ can be given by the seeker or missileborne computer. The relative velocity $v_{r}$ and the relative velocity deflection angle can be obtained by Eq. (12) and (13):

$$
\left\{\begin{array}{l}
v_{r} \cos \eta_{r}=-\dot{r} \\
v_{r} \sin \eta_{r}=r \dot{q}
\end{array}\right.
$$

Thus we have

$$
\left\{\begin{array}{l}
v_{r}=\sqrt{2+(r)^{2}} \\
\eta_{r}=\arctan \frac{r \dot{q}}{-\dot{r}}
\end{array}\right.
$$

\section{B. Analysis of Nonsingularity}

From Eq. (11), it can be seen that there is a denominator in the guidance law, so a nonsingularity analysis for the guidance law is essential. From figure 2, we can obtain the following equations.

$$
\begin{aligned}
& v_{r} \cos \eta_{r}=v_{m} \cos \left(q-\theta_{m}\right)-v_{t} \cos \left(q-\theta_{t}\right) \\
& v_{r} \sin \eta_{r}=v_{m} \sin \left(q-\theta_{m}\right)-v_{t} \sin \left(q-\theta_{t}\right)
\end{aligned}
$$

Eq. (14) multiplies by $\cos \eta_{r}$ and Eq. (15) multiplies by $\sin \eta_{r}$, than add the two equations together.

$$
v_{r}=v_{m} \cos \left(q-\theta_{m}-\eta_{r}\right)-v_{t} \cos \left(q-\theta_{t}-\eta_{r}\right)
$$

Then we have

$$
v_{m} \cos \left(q-\theta_{m}-\eta_{r}\right)=v_{r}+v_{t} \cos \left(q-\theta_{t}-\eta_{r}\right) \geq v_{r}-v_{t}
$$

It is known that the missile's velocity is much greater than the speed of the target, therefore, the missile-target relative speed must be greater than the speed of the target, which means $v_{r}-v_{t}>0$. So we can know that $v_{m} \cos \left(q-\theta_{m}-\eta_{r}\right)$ is more than zero from Eq. (17), thus, the equation of the guidance law is nonsingularity.

\section{Stability Analysis}

According to the reaching condition of sliding mode control, the Lyapunov function can be chosen as

$$
V=s^{2} / 2 \geq 0
$$

Differentiating Eq. (18) and substituting Eq. (7) and Eq. (8) into it gives

$$
\begin{aligned}
& \dot{V}=s \dot{s}=s\left(\dot{\eta}_{r}+k_{1} \dot{q}\right)=s\left[\left(k_{1}+1\right) \dot{q}-\right. \\
& \left.\frac{a_{m} \cos \left(q-\eta_{r}-\theta_{m}\right)-a_{t} \cos \left(q-\eta_{r}-\theta_{t}\right)}{v_{r}}\right]
\end{aligned}
$$

Substituting Eq. (10) into Eq. (19) yields

$$
\begin{aligned}
\dot{V} & =s\left[\frac{k_{2} \dot{r} s-\varepsilon \operatorname{sgn}(s)}{r}+\frac{a_{t} \cos \left(q-\eta_{r}-\theta_{t}\right)}{v_{r}}\right] \\
& \leq \frac{k_{2} \dot{r} s^{2}-s \varepsilon \operatorname{sgn}(s)}{r}+\frac{s M}{v_{r}} \\
& \leq \frac{k_{2} \dot{r} s^{2}}{r}-|s|\left(\frac{\varepsilon}{r}-\frac{M}{v_{r}}\right)
\end{aligned}
$$

Where $k_{2}>0, \dot{r}<0$. If $\frac{\varepsilon}{r}-\frac{M}{v_{r}}>0$ which means $\varepsilon \geq \frac{r M}{v_{r}}$, It can be ensured that $s$ is tend to zero, and the guidance system is asymptotically stable.

\section{Suppression of Chattering}

Aiming at the parameters perturbation of the control system and external disturbance the variable structure control has strong robustness, but it also has the problem of high frequency chattering. The chattering phenomenon is intrinsic to the variable structure control; it will not only reduce the control precision of the guidance system, but also destroy the system performance. As a result, how to weaken the chattering becomes the key problem of imp roving the performance of the variable structure guidance law. To weaken chattering, many scholars carry on the corresponding researches. The commonly used methods include saturated function, variable switch term and hyperbolic tangent function method [16]. In this paper, we combine the variable switch term with the saturated function to weaken the chattering, the implementation method is as follows:

The method of saturation function is to serialize the symbol function. The function can be replaced with

$$
\operatorname{sat}(s, \delta)=\frac{s}{|s|+\delta}
$$

Where $\delta>0$ and it is named boundary thickness or Chatter elimination factor [17].

The method of variable switch term is to change the equation of, improve the adaptability, to weaken the chattering. $\varepsilon$ can be described as follows to be adjusted adaptively with the change of $r$.

$$
\varepsilon=a r+b
$$

Using Eq. (21) to replace the symbol function, and Substituting Eq. (22) into Eq. (11), we can obtain the improved guidance law as 


$$
\begin{aligned}
a_{c}= & \frac{1}{\cos \left(q-\eta_{r}-\theta_{m}\right)}\left[\left(k_{1}+1\right) v_{r} \dot{q}-\right. \\
& \left.k_{2} \frac{\dot{r}}{r} v_{r} s+\frac{a r+b}{r} \frac{s}{|s|+\delta}\right]
\end{aligned}
$$

\section{Simulations}

To verify that the design of terminal Angle constraint adaptive sliding mode variable structure guidance law with terminal Angle constraint is effective, and more clearly shows that its performance, introduce the designed guidance law into the actual missile model to make a contrast simulation. In the simulation, the guidance law that the actual missile model utilizes is biased proportional guidance law with terminal angle constraint (BPN), and its proportional coefficient is 4 . The parameters of the designed sliding mode variable structure guidance law (VSG) is $k_{1}=k_{2}=3, a=0.1, b=$ 20, $\delta=0.001$. The Missile initial position coordinates are $x_{m 0}=0, y_{m 0}=1000$, and the initial velocity is $v_{m 0}=200 \mathrm{~m} / \mathrm{s}$. The target's position coordinates are $x_{t 0}=2000, y_{t 0}=0$. The expected terminal angle are $20^{\circ}, 40^{\circ}, 60^{\circ}, 80^{\circ}$, and the simulation results are as shown in Table I and Fig .2-4. Table I shows the miss distance and actual terminal angle of the two guidance law with various expected terminal angle. Fig. 2-4 show that the missile trajectory, pitching angle and overload curve of the two guidance law with the expected terminal angle that is $60^{\circ}$.

TABLE I. SIMULATION RESUL TS OF TWO ALGORITHMS

\begin{tabular}{|c|c|c|c|}
\hline $\begin{array}{c}\text { Expected terminal } \\
\text { angle }\left({ }^{\circ}\right)\end{array}$ & $\begin{array}{c}\text { Guidance } \\
\text { law }\end{array}$ & $\begin{array}{c}\text { Miss distance } \\
(\mathbf{m})\end{array}$ & $\begin{array}{c}\text { Actual terminal } \\
\text { angle } \mathbf{(}^{\circ}\end{array}$ \\
\hline \multirow{2}{*}{20} & BPN & 1.2332 & -18.7721 \\
\cline { 2 - 4 } & VSG & 0.4195 & -20.3442 \\
\hline \multirow{2}{*}{40} & BPN & 2.4382 & -33.5692 \\
\cline { 2 - 4 } & VSG & 0.7595 & -39.3379 \\
\hline \multirow{2}{*}{60} & BPN & 4.1829 & -50.7551 \\
\cline { 2 - 4 } & VSG & 1.2954 & -59.0501 \\
\hline \multirow{2}{*}{80} & BPN & 5.0846 & -69.1425 \\
\cline { 2 - 4 } & VSG & 1.4941 & -77.9897 \\
\hline
\end{tabular}

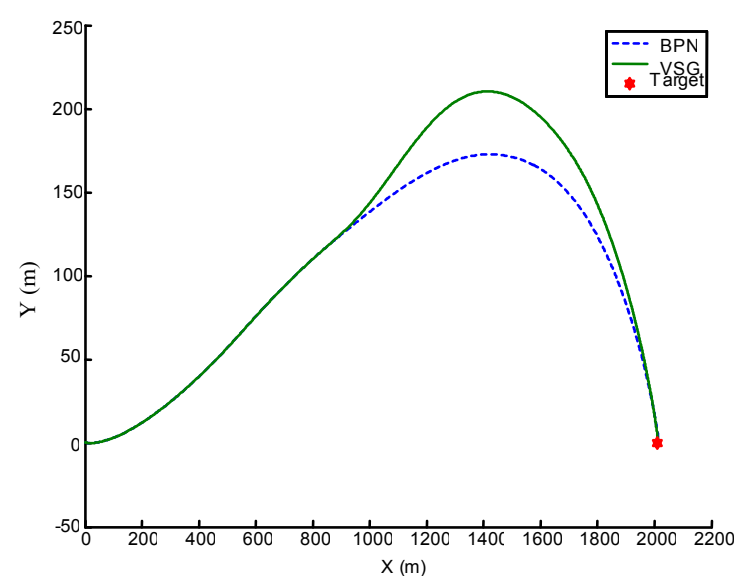

Figure 2. Trajectory of missile and target

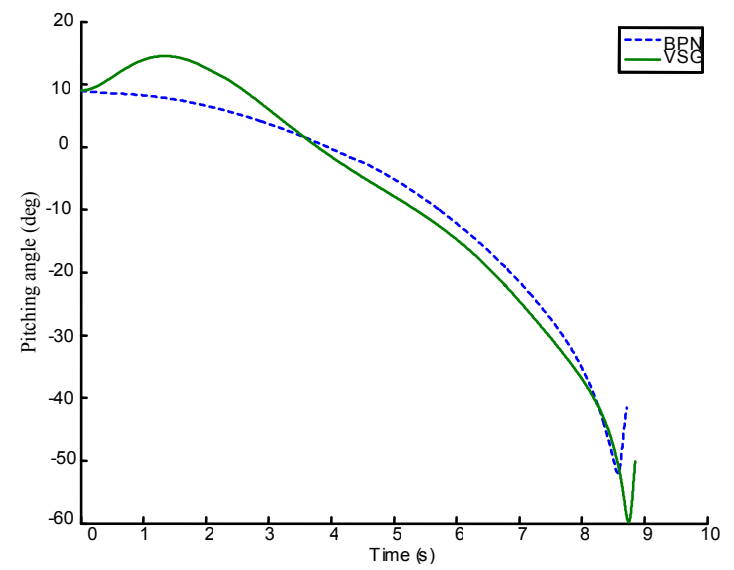

Figure 3. Chan ging of pitching angle

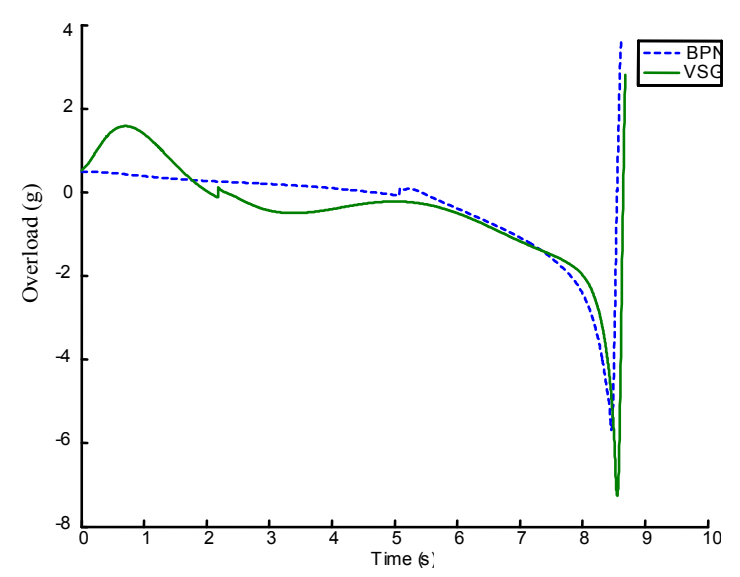

Figure 4. Chan ging of missile over load

From the above simulation results, it can be found that the guidance time of the VSG is almost as same as the BPN. Therefore, the VSG can satisfy the limitation of the guidance time. It can be seen from Table I and Fig .3 that using the VSG the minimum miss distance is $0.4195 \mathrm{~m}$; the maximum is $1.4941 \mathrm{~m}$, so the VSG can satisfy the performance requirement of the guidance weapons, such as anti-tank missile, to hit the target accurately. Under the same simulation environment, adopting the BPN the minimum miss distance is $1.2332 \mathrm{~m}$, the maximum is $5.0846 \mathrm{~m}$. For the precision guided weapons, the miss distance is obviously large, so that the weapons cannot hit the target.

Table I and Fig .4 show that, as the same expected terminal angle, the actual terminal angle by using VSG is closer to the expectation than utilizing the BPN, and the VSG has smaller angle error. Even in the case that the expected angle is $80^{\circ}$, the VSG still can satisfy the requirement, and only has a difference of $2^{\circ}$. As can be seen from the Fig .5, compared with utilizing the BPN, the missile overload by using the VSG is essentially the same, and the chattering phenomenon is suppressed well. 


\section{CONCLUSION}

In this paper, an adaptive sliding mode variable structure guidance law with terminal angle constraint has been designed based on variable structure control theory. The relative velocity deflection angle is selected as sliding mode, the Lyapunov function is used to prove the stability, and the chattering is weaken at the same time. Theoretical analys is is presented to guarantee stability and performance. The contrast simulation results are provided that the designed guidance law has greater performance than traditional guidance law in the aspect of guidance accuracy, terminal angle constraint and robustness, can greatly improve the performance of guided weapons, and its engineering applicability is well.

\section{REFERENCES}

[1] Cai H, Hu Z D, Cao Y. A survey of guidance law with terminal impact angle constraints [J]. Journal of Astronautics, 2010, 31(2):345-323.

[2] Kim M, Grider K. Terminal guidance for impact attitude angle constrained flight trajectories [J]. IEEE Transactions on Aerospace and Electronic Systems, 1973, 9(6):852-859.

[3] Ratnoo A, Ghose D. State dependent Riccati equation based guidance law for impact angle constrained trajectories [J]. Journal of Guidance, Control, and Dynamics, 2009, 32(1):320326.

[4] Zhang Q Z, Wang Z B, Tao F. Optimal guidance law design for impact with terminal angle of attack constraint [J]. Optick, 2014, 125(1):243-251.

[5] Kim B S, Lee J G, Han H S. Biased PNG law for impact with angular constraint [J]. IEEE Transactions on Aerospace and Electronic Systems, 1998, 34(1):277-288.

[6] Jeong S K, Cho S J, Kim E G. Angle constraint biased PNG [C]. The $5^{\text {th }}$ Asian Control Conference, Taejon, South Korea, 2004.
[7] Zhang $\mathrm{X}$, Lei $\mathrm{H} \mathrm{M}$, Zeng $\mathrm{H}$, et al. Adaptive proportional navigation with terminal an gu lar constraint [J]. Journal of Solid Rocket Technology, 2011, 34(6):687-692.

[8] Yoon M G. Relative circular navigation guidance for threedimensional impact angle control problem [J]. Journal of Aerospace Engineering, 2010, 23(4):300-308.

[9] $\mathrm{Hu} \mathrm{X} \mathrm{J,} \mathrm{Huang} \mathrm{X} \mathrm{M.} \mathrm{Circular} \mathrm{trajectory} \mathrm{guidance} \mathrm{law} \mathrm{with}$ terminal constraints of impact point and impact angle [J]. Journal of Astronautics, 2012, 33(5):562-569.

[10] Zhao Y, Sheng Y Z. Sliding mode control based guidance law with impact angle constraint $[\mathrm{J}]$. Chinese Journal of Aeronautics, 2014, 27(1):145-152.

[11] Wu P, Yang M. Variable structure guidance law with terminal attack angle constraint [J]. Journal of Solid Rocket Technology, 2008, 31(2):116-120.

[12] Harl N, Balakrishnan S N. Impact time and angle guidance with sliding mode control [J]. IEEE Transactions on Control Systems Technology, 2012, 20(6):1436-1449.

[13] Guo H W, Lin W S, Liu Ming-jun. A variable structure guidance law based on relative velocity deflection angle $[\mathrm{J}]$. Journal of Astronautics, 2001, 22(3):33-37.

[14] Lin J X, Zhao Y T, Hu Y A. Design of a variable structure terminal guidance law based on relative velocity deflection angle $[\mathrm{J}]$. Journal of Naval Aeronautical and Astronautical University, 2009, 24(6):646-650.

[15] Gao F, Tang S J, Shi J, et al. An improved adaptive slidingmode navigation guidance law with variable structure $[\mathrm{J}]$. Journal of Ballistics, 2013, 25(3): 18-23.

[16] Levant A. Chattering Analysis [J]. IEEE Transactions on Automatic Control, 2010, 55(6):1380-1389.

[17] Jia Q Z, Liu Y S, Liu Z Z. Variable-structure back stepping guidance law with terminal an gular constraint for video guided penetrating bomb [J]. Journal of Astronautics, 2008, 29(1): 280-214. 